\title{
Application of Electromyography in Rehabilitation Devices
}

\author{
Bhardwaj S, Khan AA* and Muzammil M \\ Department of Mechanical Engineering, Aligarh Muslim University, Aligarh (UP), India
}

*Corresponding author: Abid Ali Khan, Department of Mechanical Engineering, Aligarh Muslim University, Aligarh (UP), India, Email: abid.khan.me@amu.ac.in

\section{Research Article}

Volume 4 Issue 4

Received Date: May 25, 2020

Published Date: July 02, 2020

DOI: $10.23880 /$ eoij-16000242

\section{Abstract}

With the increase in disability rate as well as rise in the elderly population, there is a need for a much prompt and advance methods to deliver the rehabilitation and assistive services to the patients. One important element in this context is the robotics rehabilitation devices. The therapeutic alliance is an important factor that needs to be addressed for these robotic devices where user compliance is still a broad field to be explored. Bio-signals have shown a promising approach in improving user physical and cognitive integration with these devices. Electromyogram (EMG) is one such bio-signal, originating from the peripheral nervous system, which is capable of interpreting the motion intent of the user. In the present study, the usability of EMG is shown for its feasibility in the context of rehabilitation devices. Method for acquisition, processing and classification of EMG has been discussed.

Keywords: Electromyography; Data Acquisition System; Classification

\section{Introduction}

Achieving human machine compliance is the main objective in rehabilitation robotics. Not only does having a good compliance helps to achieve a better assistance in activities of daily living, but in turn also aids in faster recovery of the patients [1]. Older generations of rehabilitation robots rely on traditional user interfaces such as joysticks, etc., which are generally difficult for the user to control. Therefore, a more advanced user-machine interface is sought to learn the intention of the user and therefore control/manipulate the parameters according to the patients' intention and their recovery timeline [2].

Bio-signals plays a crucial role in measuring the human intention towards movement, and hence the measurement of assistance required. Electromyography signal (EMG), is a non-stationary signal which contains rich information about human intent to motion [3]. Moreover, EMG generation occurs approximately 20 to $80 \mathrm{~ms}$ before actual limb movement takes place [4]. With these advantages, EMG has found its usability in biofeedback applications concerning both neurological and musculoskeletal rehabilitation [5].
However, it is important to first process the acquired raw EMG signals into meaningful features, which are then classified to predict the desired motion [6].

The objective of this paper is to demonstrate the feasibility of EMG in developing rehabilitation application. Sit to stand (STS), which is a task of daily living, was considered for the present study. STS task poses a significant challenge for the people with lower limb weakness. The geriatric population and people with lower limb disabilities can benefit from the provision of assistive aids, and providing EMG control will make the aid autonomous for the user. With this notion, the acquisition, processing and classification of EMG data have been discussed regarding the STS task.

\section{Methodology}

\section{Data Acquisition}

EMG of vastus lateralis (VL) of both the legs (Left-VL and Right-VL) were recorded from five healthy participants (with their consent and approval from the ethics committee). Bipolar surface electrodes (SX230, Biometrics Ltd.) were used 
to measure the EMG signal. The electrodes were connected to a DataLINK signal conditioning unit (DLK900, Biometrics Ltd.) that outputs an analog two channel signal to the NI6211 DAQ (National Instruments Ltd.). The DAQ system was communicated with the PC using a custom build LabVIEW program. Grounding electrode was placed at lunate bone of the left wrist for reducing high frequency DC artifacts. The sEMG data was sampled at $1000 \mathrm{~Hz}$.

\section{Processing and Feature Extraction}

The EMG signal was high pass filtered using a Butterworth 2nd order IIR filter with a $20 \mathrm{~Hz}$ cut off frequency and $50 \mathrm{~Hz}$ notch filter. Root mean square (RMS) was evaluated as the EMG feature. A Triangle-Bartlett windowing RMS filter was designed to calculate the RMS of the filtered EMG signal over a segment length of 500 samples.

\section{Classification}

Naive-Bayes model was used to classify the four different phases of STS task. The phases were determined based upon the angular deviation of the trunk and knee [7]. The four phases were: Phase I (P1) - sitting phase, where the participant sits comfortably on the stool; Phase II (P2) - intention to stand from sitting posture; Phase III (P3) Transition phase; and Phase IV (P4) - standing phase. The training was performed on the $70 \%$ of the data while testing was performed on the rest $30 \%$ of the data.

\section{Result and Discussion}

A sample recording for a participant showing the computed RMS is shown in Figure 1. The phase P1, P2, P3 and $\mathrm{P} 4$ consisted of $12.9 \%, 13.0 \%, 66.8 \%$ and $7.4 \%$ of the STS task. Peak EMG RMS of VL was observed during P3 phase where the actual knee extension took place.

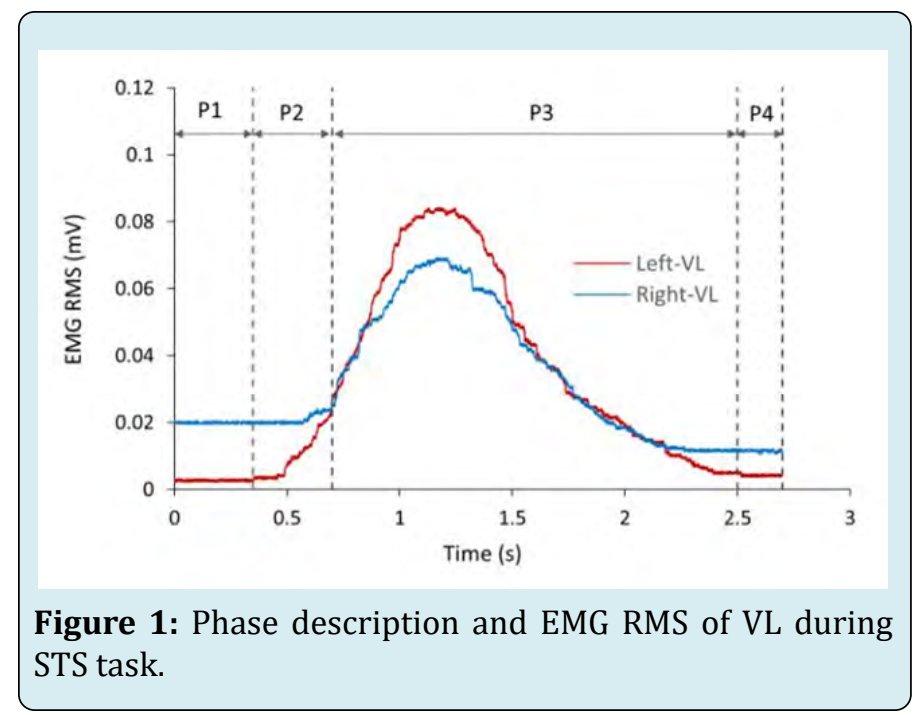

Figure 2 shows the confusion matrix for the NaiveBayes model used to classify the STS phases. The diagonal elements represent the degree of correctly predicted classes. The confusion is expressed by the falsely classified diagonal elements. Average true positive rate for the model was 92.4 $\%$. The highest classification error was reported for P2 i.e. the intention detection phase, when the EMG activity was just started without actual movement in the knees.

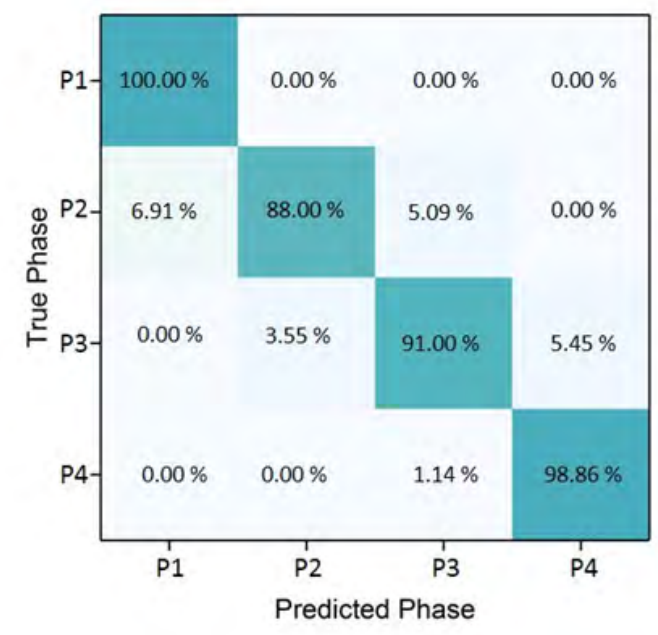

Figure 2: Normalized confusion matrix for Naive-Bayes classification rate.

The proposed model shows a unimodal approach for EMG classification. P2 is the most important phase concerning the measurement of intent in performing STS task. The classification accuracy can be further improved by considering a multimodal approach. Instead of only using EMG, a combination of EMG with body kinematics may improve the classification accuracy and precision [7].

\section{Conclusion}

Electromyography is frequently used to measure the human intentions in performing movements. The present article gives an overview on how the EMG signals are acquired, processed and classified for use in rehabilitation/ assistive applications. Naive-Bayes model predicted the overall classification accuracy of $92.4 \%$ for STS task.

\section{References}

1. Wilmart R, Garone E, Innocenti B (2019) The use of robotics devices in knee rehabilitation: a critical review. Muscles Ligaments Tendons Journal 9(1): 21-48.

2. Rechy-Ramirez EJ, Hu H (2015) Bio-signal based control 
in assistive robots: a survey. Digital Communications and Networks 1(2): 85-101.

3. Fleischer C, Reinicke C, Hommel G (2005) Predicting the intended motion with EMG signals for an exoskeleton orthosis controller. International Conference on Intelligent Robots and Systems, Canada.

4. Norman RW, Komi PV (1979) Electromechanical delay in skeletal muscle under normal movement conditions. Acta Physiologica Scandinavica 106(3): 241-248.

5. Giggins OM, Persson UM, Caulfield B (2013) Biofeedback in rehabilitation. Journal of neuroengineering and rehabilitation 10: 1-11.

6. Bhardwaj S, Khan AA, Muzammil M (2016) Electromyography in physical rehabilitation: a review. National Conference on Mechanical Engineering-Ideas, Innovations \& Initiatives, India.

7. Bhardwaj S, Khan AA, Muzammil M (2019) Lower limb rehabilitation using multimodal measurement of sitto-stand and stand-to-sit task. Disability Rehabilitation Assistive Technology 10: 1-8. 